\title{
QCD Coupling from a Nonperturbative Determination of the Three-Flavor $\Lambda$ Parameter
}

\author{
Mattia Bruno, ${ }^{1}$ Mattia Dalla Brida, ${ }^{2}$ Patrick Fritzsch, ${ }^{3}$ Tomasz Korzec, ${ }^{4}$ Alberto Ramos, ${ }^{3}$ Stefan Schaefer, ${ }^{5}$ \\ Hubert Simma, ${ }^{5}$ Stefan Sint, ${ }^{6}$ and Rainer Sommer ${ }^{5,7}$
}

(ALPHA Collaboration)

\begin{abstract}
${ }^{1}$ Physics Department, Brookhaven National Laboratory, Upton, New York 11973, USA
${ }^{2}$ Dipartimento di Fisica, Università di Milano-Bicocca and INFN, Sezione di Milano-Bicocca, Piazza della Scienza 3, 20126 Milano, Italy

${ }^{3}$ Theoretical Physics Department, CERN, 1211 Geneva 23, Switzerland

${ }^{4}$ Department of Physics, Bergische Universität Wuppertal, Gaußstrasse 20, 42119 Wuppertal, Germany

${ }^{5}$ John von Neumann Institute for Computing (NIC), DESY, Platanenallee 6, 15738 Zeuthen, Germany

${ }^{6}$ School of Mathematics and Hamilton Mathematics Institute, Trinity College Dublin, Dublin 2, Ireland

${ }^{7}$ Institut für Physik, Humboldt-Universität zu Berlin, Newtonstrasse 15, 12489 Berlin, Germany
\end{abstract}

(Received 13 June 2017; published 8 September 2017)

\begin{abstract}
We present a lattice determination of the $\Lambda$ parameter in three-flavor QCD and the strong coupling at the $Z$ pole mass. Computing the nonperturbative running of the coupling in the range from 0.2 to $70 \mathrm{GeV}$, and using experimental input values for the masses and decay constants of the pion and the kaon, we obtain $\Lambda \frac{(3)}{\mathrm{MS}}=341(12) \mathrm{MeV}$. The nonperturbative running up to very high energies guarantees that systematic effects associated with perturbation theory are well under control. Using the four-loop prediction for $\Lambda \frac{(5)}{\mathrm{MS}} / \Lambda_{\overline{\mathrm{MS}}}^{(3)}$ yields $\alpha \frac{(5)}{\mathrm{MS}}\left(m_{Z}\right)=0.11852(84)$.
\end{abstract}

DOI: 10.1103/PhysRevLett.119.102001

Introduction.-An essential input for theory predictions of high energy processes, in particular for phenomenology at the LHC [1-4], is the QCD coupling $\alpha_{s}(\mu)=\bar{g}_{s}^{2}(\mu) /(4 \pi)$ at energy scales $\mu \sim m_{Z}$ and higher. In this work we present a subpercent determination of the strong coupling at the $Z$ pole mass using the masses and decay constants of the pion and kaon as experimental input and lattice QCD as a computational tool.

Perturbation theory (PT) predicts the energy dependence of the coupling as

$\bar{g}_{s}^{2}(\mu) \stackrel{\mu \rightarrow \infty}{\sim} \frac{1}{2 b_{0} \log \left(\mu / \Lambda_{s}\right)+\left(b_{1} / b_{0}\right) \log \log \left(\mu / \Lambda_{s}\right)}+\cdots$

in terms of known positive coefficients, $b_{0,1}$, and a single parameter, $\Lambda_{s}$, which can also serve as the nonperturbative scale of the theory. The label $s$, called scheme, summarizes all details of the exact definition of $\bar{g}_{s}$. Conventionally one chooses the so-called $s=\overline{\mathrm{MS}}$ scheme [5], but $\Lambda$ parameters in different schemes can be exactly related with a one-loop computation [6].

Published by the American Physical Society under the terms of the Creative Commons Attribution 4.0 International license. Further distribution of this work must maintain attribution to the author(s) and the published article's title, journal citation, and DOI.
Our computation of $\alpha_{\overline{\mathrm{MS}}}$ is based on a determination of the three-flavor $\Lambda$ parameter. To outline the steps of our determination, we write

$$
\Lambda \frac{(3)}{\mathrm{MS}}=\frac{\Lambda_{\mathrm{MS}}^{(3)}}{\mu_{\mathrm{PT}}} \times \frac{\mu_{\mathrm{PT}}}{\mu_{\mathrm{had}}} \times \frac{\mu_{\mathrm{had}}}{f_{\pi \mathrm{K}}} \times f_{\pi \mathrm{K}}^{\mathrm{PDG}} .
$$

As experimental input we use the Particle Data Group (PDG) values [7] for the following combination of decay constants:

$$
f_{\pi \mathrm{K}} \equiv \frac{1}{3}\left(2 f_{\mathrm{K}}+f_{\pi}\right)=147.6 \mathrm{MeV} .
$$

The key elements are then the determination of the ratio of scales $\mu_{\mathrm{PT}} / \mu_{\text {had }}$ and the ratio $\mu_{\text {had }} / f_{\pi \mathrm{K}}$, i.e., our hadronic scale in units of $f_{\pi \mathrm{K}}$. Both computations are performed in a fully nonperturbative way.

By choosing a large enough scale $\mu_{\mathrm{PT}}$ and including higher orders of PT in Eq. (1), the ratio $\Lambda_{\overline{\mathrm{MS}}}^{(3)} / \mu_{\mathrm{PT}}$ can be determined with negligible errors.

With $N_{f}>2$ flavors, so far a single work [8] contains such a computation with all steps, including the connection of low energy $\mu_{\text {had }}$ to large $\mu_{\mathrm{PT}}$, using numerical simulations and a step scaling strategy. This strategy, developed by the ALPHA Collaboration [9-12], suppresses the systematic errors from the use of PT.

Here, we put together (and briefly review) the first factor in Eq. (2) and our recent significant improvements in 
TABLE I. Summary of various scales used in this work.

\begin{tabular}{clc}
\hline \hline Scale definition & \multicolumn{1}{c}{ Purpose } & $\mu / \mathrm{GeV}$ \\
\hline$\mu_{\mathrm{PT}}=16 \mu_{0}$ & $\begin{array}{l}\text { Matching with the asymptotic } \\
\text { perturbative behavior }\end{array}$ & $\approx 70$ \\
$\bar{g}_{\mathrm{SF}}^{2}\left(\mu_{0}\right)=2.012$ & $\begin{array}{l}\text { Nonperturbative matching } \\
\text { between the GF and SF schemes }\end{array}$ & $\approx 4$ \\
$\bar{g}_{\infty}^{2}\left(\mu_{\mathrm{ref}}^{\star}\right)=1.6 \pi^{2}$ & $\begin{array}{l}\text { Setting scale in physical units } \\
\text { by experimental value for } f_{\pi \mathrm{K}}\end{array}$ & $\approx 0.5$ \\
$\bar{g}_{\mathrm{GF}}^{2}\left(\mu_{\mathrm{had}}\right)=11.31$ & $\begin{array}{l}\text { Matching between GF scheme } \\
\text { and infinite-volume scheme }\end{array}$ & $\approx 0.2$ \\
\hline \hline
\end{tabular}

statistical and systematic precision in the second one $[13,14]$, and finally add the missing third one.

QCD with $N_{f}=3$ is the phenomenologically relevant effective theory at energies $E<m_{\text {charm }}$ with small $[15,16]$ corrections of order $\left(E / m_{\text {charm }}\right)^{2}$. However, for theory predictions of high energy processes, with $E \sim m_{Z}$ and higher, the five- and six-flavor theories are needed. Fortunately, the ratios $\Lambda_{\overline{\mathrm{MS}}}^{\left(N_{f}\right)} / \Lambda_{\mathrm{MS}}^{(3)}, N_{f}=4,5,6$ are known to very high order in PT, and successive order contributions decrease rapidly. This enables us to convert our $\Lambda_{\overline{\mathrm{MS}}}^{(3)}$ to precise values for $\alpha_{\overline{\mathrm{MS}}}^{(5)}\left(m_{Z}\right)$ and $\alpha_{\overline{\mathrm{MS}}}^{(6)}(1.508 \mathrm{TeV})$, which can be used for high energy phenomenology. Further below, we will critically discuss the use of PT in this step.

Strategy.-A nonperturbative definition of a coupling is easily given. Take a short-distance QCD observable, depending on fields concentrated within a 4D region of Euclidean space of linear size $R=1 / \mu$ and with a perturbative expansion

$$
\mathcal{O}_{s}(\mu)=k \bar{g}_{\overline{\mathrm{MS}}}^{2}(\mu)\left[1+c_{1}^{s} \bar{g}_{\overline{\mathrm{MS}}}^{2}(\mu)+\cdots\right] .
$$

Then the nonperturbative coupling,

$$
\bar{g}_{s}^{2}(\mu) \equiv \mathcal{O}_{s}(\mu) / k=\bar{g}_{\overline{\mathrm{MS}}}^{2}(\mu)+c_{1}^{s} \bar{g}_{\overline{\mathrm{MS}}}^{4}(\mu)+\cdots,
$$

runs with $\mu$. This property also allows us to define scales $\mu$ by fixing $\bar{g}_{s}^{2}(\mu)$ to particular values (see Table I). However, there is a challenge to reach the asymptotic region of small $\bar{g}_{s}^{2}(\mu)$, where Eq. (1) is useful and its corrections can be controlled, using lattice simulations.

Challenge.-Numerical computations involve both a discretization length, the lattice spacing $a$, and a total size of the system $L$, that is simulated. For standard observables, control over finite volume effects of order $\exp \left(-m_{\pi} L\right)$ requires $L$ to be several $\mathrm{fm}$. At the same time, one needs to suppress discretization errors and should extrapolate $(a \mu)^{2} \rightarrow 0$ at fixed $\mu$. The necessary restrictions

$$
L \gg 1 / m_{\pi}, 1 / a \gg \mu \Rightarrow L / a \gg \mu / m_{\pi}
$$

translate into very large lattices. Figure 1 displays the region in $\alpha(\mu)$ vs $(a \mu)^{2}$ for the range $a \geq 0.04 \mathrm{fm}$ which can be realized nowadays in large volumes $\left(m_{\pi} L \geq 4\right)$. This shaded region is quite far from small coupling and small $(a \mu)^{2}$.

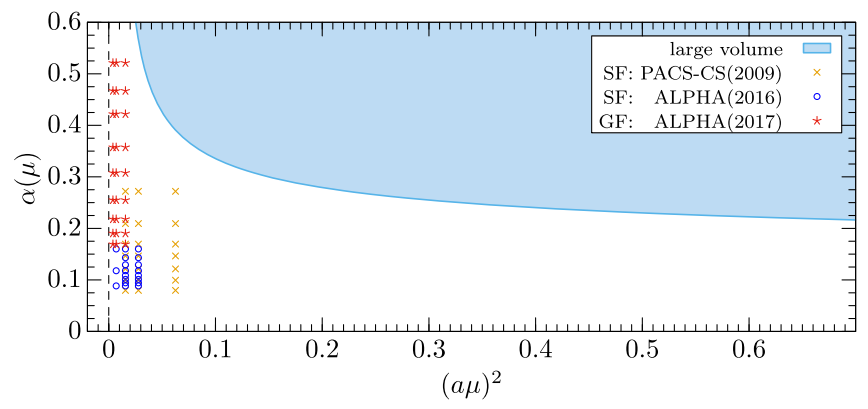

FIG. 1. The shaded area shows the $a>0.04 \mathrm{fm}$ region of large volume results which dominate the present PDG and FLAG estimates [7,17] of $\alpha_{\overline{\mathrm{MS}}}(\mu)$. The boundary of the shaded area is determined by $\alpha_{\overline{\mathrm{MS}}}(\mu)$ evaluated at two-loop order for $N_{f}=3$ and $\Lambda \frac{(3)}{\mathrm{MS}}=341 \mathrm{MeV}$. The $x$ coordinate is then obtained by setting $a=0.04 \mathrm{fm}$, the smallest value of lattice spacings reached in the large volume simulations entering [7,17]. The data points on the left are finite-size scaling computations $[8,13,14]$.

Finite-size schemes.-The way out has long been known [9,12]. One may identify $R=L=1 / \mu$ by choosing $\mathcal{O}_{s}$ to depend only on the scale $L$, not on any other ones. Finitesize effects become part of the observable rather than one of its uncertainties. Equation (6) is then relaxed to

$$
L / a \gg 1,
$$

such that $L / a=10-50$ is sufficient.

Different scales $\mu$ are then connected by the step scaling function

$$
\left.\sigma(u) \equiv \bar{g}_{s}^{2}(\mu / 2)\right|_{\bar{g}_{s}^{2}(\mu)=u} .
$$

It describes scale changes by discrete factors of 2 , in contrast to the $\beta$ function which is defined by infinitesimal changes. For a chosen value of $u, \sigma(u)$ can be computed by determining $\bar{g}^{2}$ on lattices of size $L / a$ and $2 L / a$ and performing an extrapolation $a \rightarrow 0$ at $L=1 / \mu$, fixed through $\bar{g}^{2}(\mu)=u$. In fact, in the process also the $\beta$ function can be computed as long as $\sigma(u)$ is a smooth function of $u$. A recent detailed description of step scaling is given in Ref. [18].

Running coupling in the three-flavor theory between $200 \mathrm{MeV}$ and $100 \mathrm{GeV}$.-We impose Schrödinger Functional (SF) boundary conditions on all fields $[19,20]$, i.e., Dirichlet boundary conditions in Euclidean time at $x_{0}=0, L$, and periodic boundary conditions in space with period $L$. With this choice, one can define different renormalized couplings in the massless theory $[14,19,21]$ and complications with perturbation theory [22] are avoided.

First, we consider the SF coupling [19,23], $\bar{g}_{\mathrm{SF}}(\mu)$, which measures how the system reacts to a particular change of the boundary conditions. When computed by Monte Carlo methods, this coupling has a statistical uncertainty that scales as $\Delta_{\mathrm{stat}} \bar{g}_{\mathrm{SF}}^{2} \sim \bar{g}_{\mathrm{SF}}^{4}$, leading to good precision at high energies. Moreover, its $\beta$ function is known to NNLO 
[24,25]. These two properties make it an ideal choice to match with the asymptotic perturbative regime of QCD.

Second, one can use the gradient flow (GF) to define renormalized couplings [26]. For this purpose, one introduces a flow time $t \geq 0$ and defines the flow field $B_{\mu}(t, x)$ as the solution of the gradient flow equation

$$
\begin{aligned}
\partial_{t} B_{\mu}(t, x) & =D_{\nu} G_{\nu \mu}(t, x), \\
G_{\mu \nu}(t, x) & =\partial_{\mu} B_{\nu}-\partial_{\nu} B_{\mu}+\left[B_{\mu}, B_{\nu}\right],
\end{aligned}
$$

with the initial value $B_{\mu}(0, x)=A_{\mu}(x)$ given in terms of the original gauge field. The flow time $t$ has the dimension of a squared length and is a new external scale which can be chosen at will. For $t>0$, fluctuations of $A_{\mu}(x)$ at length scales much smaller than $\sqrt{8 t}$ are suppressed in the flow field $B_{\mu}(t, x)$. This smoothing property implies that gauge invariant composite fields made out of $B_{\mu}(t, x)$ are finite [27] and can be used to define renormalized couplings. In particular, in infinite volume such a coupling can be defined as

$$
\bar{g}_{\infty}^{2}(\mu)=\frac{16 \pi^{2}}{3} \times\left. t^{2}\langle E(t)\rangle\right|_{\mu=1 / \sqrt{8 t}},
$$

in terms of the action density [26] $E(t)=\frac{1}{4} G_{\mu \nu}^{a}(t, x) G_{\mu \nu}^{a}(t, x)$.

In finite volume the coupling $\bar{g}_{\mathrm{GF}}^{2}(\mu)$ is defined by imposing a fixed relation, $\sqrt{8 t}=c L$, between the flow time and the volume $[21,28]$. Details can be found in the original work [14]. Since the statistical precision is generally good and scales as $\Delta_{\text {stat }} \bar{g}_{\mathrm{GF}}^{2} \sim \bar{g}_{\mathrm{GF}}^{2}$, this coupling is well suited at low energies.

In order to exploit the advantages of both finite-volume schemes, we use the GF scheme at low energies, between $\mu_{\text {had }}$ and $\mu_{0}$. There we switch nonperturbatively to the SF scheme (see Fig. 2). Then we run up to $\mu_{\mathrm{PT}}$. In this way, we connected hadronic scales to $\mu_{\mathrm{PT}}[13,14]$, cf. Table I.

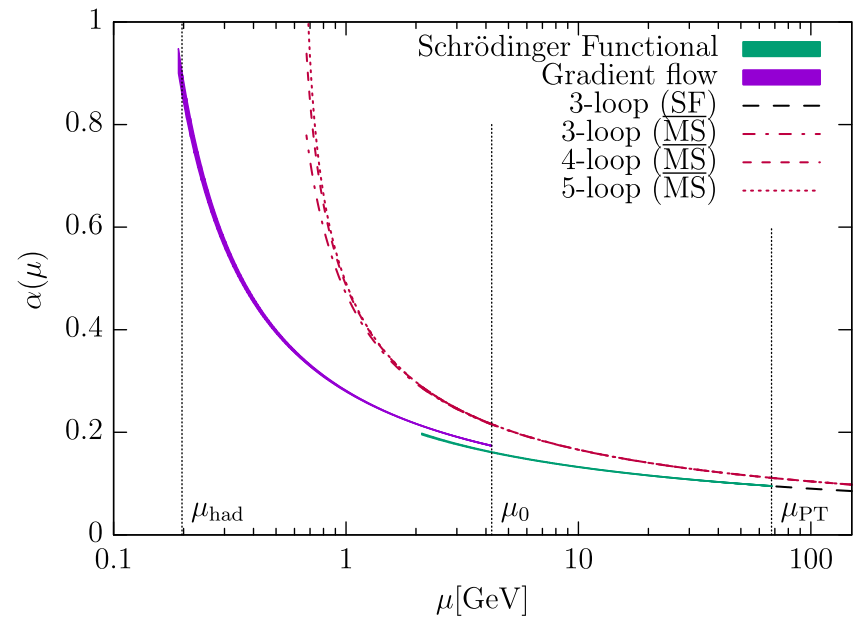

FIG. 2. Running couplings of $N_{f}=3$ QCD from integrating the nonperturbative $\beta$ functions in the $\mathrm{SF}$ and $\mathrm{GF}$ schemes $[13,14]$. They are matched nonperturbatively by defining $\bar{g}_{\mathrm{SF}}^{2}\left(\mu_{0}\right)=2.012$ and computing $\bar{g}_{\mathrm{GF}}^{2}\left(\mu_{0} / 2\right)=2.6723(64)$.
TABLE II. Scale ratios and values of the coupling determined from nonperturbative running from $\mu_{\text {had }}$ to $\mu_{0} / 2$ in the GF and from $\mu_{0}$ to $\mu_{\mathrm{PT}}$ in the SF scheme.

\begin{tabular}{llll}
\hline \hline $\bar{g}_{\mathrm{GF}}^{2}\left(\mu_{\mathrm{had}}\right)$ & $\bar{g}_{\mathrm{SF}}^{2}\left(\mu_{\mathrm{PT}}\right)$ & $\mu_{\mathrm{PT}} / \mu_{\text {had }}$ & $\Lambda_{\frac{(3)}{\mathrm{MS}} / \mu_{\mathrm{had}}}$ \\
\hline 11.31 & $1.193(5)$ & $349.7(6.8)$ & $1.729(57)$ \\
10.20 & $1.193(5)$ & $322.2(6.3)$ & $1.593(53)$ \\
\hline \hline
\end{tabular}

In Table II we show our intermediate results for $\bar{g}_{\mathrm{SF}}^{2}\left(\mu_{\mathrm{PT}}\right)$ and $\mu_{\mathrm{PT}} / \mu_{\text {had }}$ for two choices of a typical hadronic scale $\mu_{\text {had }}$ of a few hundred MeV. (In Ref. [14] only $\mu_{\text {had, }, 1}$ was considered. Here we extend the analysis to $\mu_{\text {had, }, 2}$ in order to have an additional check of our connection of large and small volume physics.) In addition, we give $\Lambda \frac{(3)}{\mathrm{MS}} / \mu_{\text {had }}$, obtained by the NNLO perturbative asymptotic relation and the exact conversion to the $\overline{\mathrm{MS}}$ scheme. We have verified that the systematic uncertainty $\sim \alpha^{2}\left(\mu_{\mathrm{PT}}\right)$ and power corrections $\sim\left(\Lambda / \mu_{\mathrm{PT}}\right)^{k}$ from this limited use of perturbation theory at scales above $\mu_{\mathrm{PT}}$ are negligible compared to our statistical uncertainties $[13,29]$.

Connection to the hadronic world.-The second key element is the nonperturbative determination of $\mu_{\text {had }}$ in units of the experimentally accessible $f_{\pi \mathrm{K}}$. Our determination is based on CLS ensembles [30] of $N_{f}=3$ QCD with $m_{u}=m_{d} \equiv \hat{m}$ in large volume. It is convenient to define a scale $\mu_{\text {ref }}$ by the condition

$$
\bar{g}_{\infty}^{2}\left(\mu_{\text {ref }}\right)=1.6 \pi^{2} \approx 15.8,
$$

and trajectories in the (bare) quark mass plane $\left(\hat{m}, m_{s}\right)$ by keeping the dimensionless ratio

$$
\phi_{4}=\left(m_{\mathrm{K}}^{2}+m_{\pi}^{2} / 2\right) / \mu_{\mathrm{ref}}^{2}
$$

constant. (Note that $\mu_{\text {ref }}$ is defined ensemble by ensemble, and therefore it is a function of the quark masses. Instead of $\mu_{\text {ref }}$, it is customary in the lattice literature to quote $\sqrt{8 t_{0}}=$ $1 / \mu_{\text {ref }}$ [26].) Moreover, we define a reference scale $\mu_{\text {ref }}^{\star}$ at the symmetric point $\left(m_{u}=m_{d}=m_{s}\right)$ by

$$
\left.\mu_{\text {ref }}^{\star} \equiv \mu_{\text {ref }}\right|_{\phi_{4}=1.11, m_{u}=m_{d}=m_{s}} .
$$

The requirement that the $\phi_{4}=$ constant trajectory passes through the physical point, defined by

$$
m_{\pi}^{2} / f_{\pi \mathrm{K}}^{2}=0.8341, \quad m_{\mathrm{K}}^{2} / f_{\pi \mathrm{K}}^{2}=11.21,
$$

results in $\phi_{4}=1.11(2)$ in the continuum limit [31] and motivates the particular choice in Eq. (13).

Since the combination $f_{\pi \mathrm{K}}$ has a weak and well understood dependence on the pion mass along trajectories with constant $\phi_{4}$, a precise extrapolation from the symmetric point to the physical point can be performed [31,32], see Fig. 3. Continuum extrapolations with four lattice spacings, $0.05 \mathrm{fm} \lesssim a \lesssim 0.09 \mathrm{fm}$, together with the PDG value of Eq. (3), yield [31] 


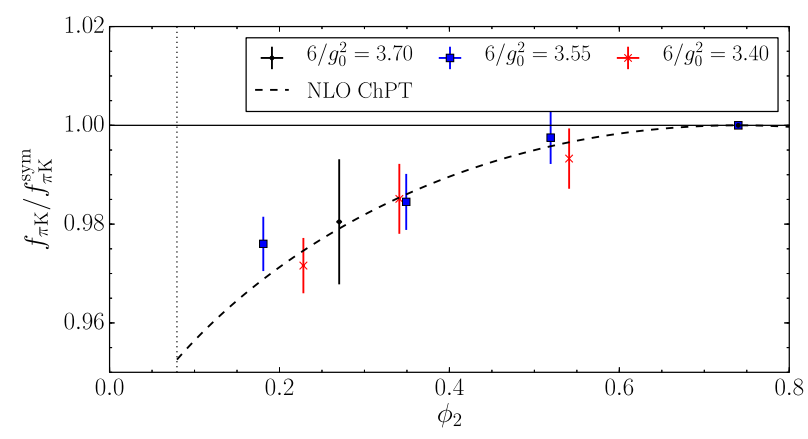

FIG. 3. Dependence of $f_{\pi \mathrm{K}}$ at $\phi_{4}=1.11$ on the pion mass through $\phi_{2}=m_{\pi}^{2} / \mu_{\text {ref }}^{2}$ [31]. We normalized to $f_{\pi \mathrm{K}}^{\mathrm{sym}}$ at the symmetric point $m_{u}=m_{d}=m_{s}$. The ratio follows the parameter-free prediction of NLO chiral PT.

$$
\mu_{\mathrm{ref}}^{\star}=478(7) \mathrm{MeV} \text {. }
$$

Note that $\mu_{\text {ref }}^{\star}$ is defined at a point with unphysical quark masses, where finite-size effects are smaller and simulations are easier than close to the physical point. This allows us to include in the following analysis a CLS ensemble at a fifth lattice spacing, $a \approx 0.039 \mathrm{fm}$.

For the determination of $\mu_{\text {ref }}^{\star} / \mu_{\text {had }}$, we need pairs of values $a \mu_{\text {had }}$ and $a \mu_{\text {ref }}^{\star}$ at the same value of $a$. This requires either an interpolation of the data for $a \mu_{\text {had }}$, or an interpolation of the data for $a \mu_{\mathrm{ref}}^{\star}$. We denote these two options as set $A$ and $B$, respectively.

The dimensionless ratio $\mu_{\text {ref }}^{\star} / \mu_{\text {had }}$ can then be extrapolated to the continuum as shown in Fig. 4. Extrapolations, linear in $a^{2}$ dropping data above $\left(a \mu_{\text {ref }}^{\star}\right)^{2}=0.07$ with either set A or B, are fully compatible. They are also stable under changes in the number of points used to extrapolate and the particular functional form. These stabilities are expected since our smallest lattice spacing is $a \approx 0.039 \mathrm{fm}$. We repeat the computation of $\mu_{\text {ref }} / \mu_{\text {had }}$ for two different values of $\mu_{\text {had }}$ (see Fig. 4). Tables of the various numbers that enter

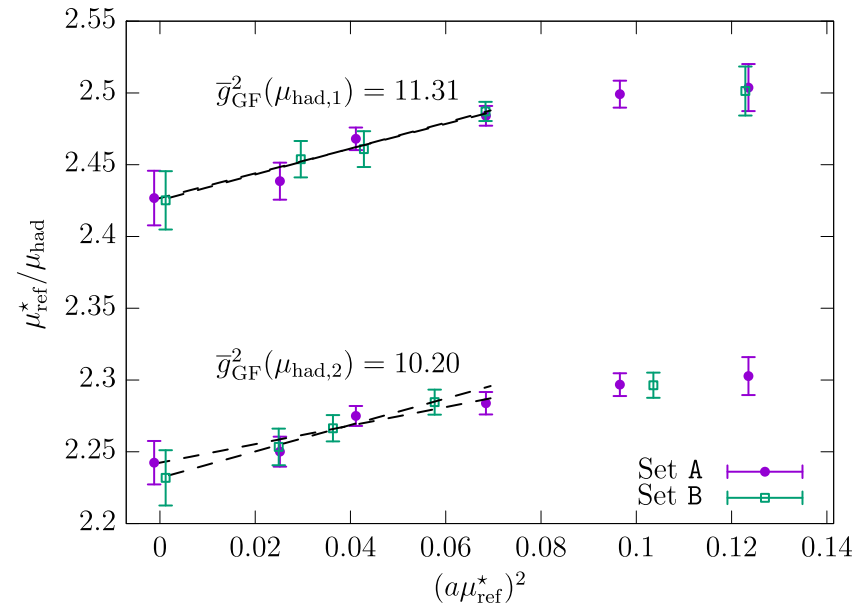

FIG. 4. Continuum extrapolations described in the text. Extrapolated values are shown in proximity of $a=0$. and further details can be found in the Supplemental Material [33], which includes Refs. [34-43].

As our final estimates we take set B, which has somewhat larger errors, and obtain

$$
\begin{array}{rlrl}
\frac{\mu_{\text {ref }}^{\star}}{\mu_{\text {had }, 1}}=2.428(18), & & \frac{\Lambda_{\mathrm{MS}}^{(3)}}{\mu_{\text {ref }}^{\star}}=0.712(24), \\
\frac{\mu_{\text {ref }}^{\star}}{\mu_{\text {had }, 2}}=2.233(17), & \frac{\Lambda_{\mathrm{MS}}^{(3)}}{\mu_{\text {ref }}^{\star}}=0.713(24) .
\end{array}
$$

The close agreement in $\Lambda \frac{(3)}{\mathrm{MS}} / \mu_{\text {ref }}^{\star}$ is reassuring. With Eq. (15) we arrive at our central result

$$
\Lambda_{\mathrm{MS}}^{(3)}=341(12) \mathrm{MeV} \text {. }
$$

It has a remarkable precision given that we ran the couplings nonperturbatively up to about $70 \mathrm{GeV}$ and only then used perturbation theory.

$\Lambda$ parameters and couplings of five- and six-flavor theories.-By itself our $\Lambda^{(3)}$ is of limited phenomenological use. The three-flavor effective field theory (EFT) is valid for energies below $m_{\text {charm }}=1.28 \mathrm{GeV}$. There perturbation theory cannot be expected to be precise.

However, QCD ${ }^{\left(N_{f}\right)}$, the $N_{f}$-flavor effective theory, can be matched to $\operatorname{QCD}^{\left(N_{f}+1\right)}$ and one can eventually arrive at $\mathrm{QCD}^{(6)}$ [44]. This matching relates the couplings such that the low(er) energy EFT agrees with the (more) fundamental one up to power law corrections. These $O\left(\Lambda^{2} / m_{h}^{2}\right)$ corrections can only be studied nonperturbatively. They are very small already for $m_{h}=m_{\text {charm }}[15,16]$.

Ignoring $1 / m_{h}^{2}$ effects, matching means

$$
\bar{g}^{\left(N_{f}\right)}(\mu)=\bar{g}^{\left(N_{f}+1\right)}(\mu) \times \xi\left(\bar{g}^{\left(N_{f}+1\right)}(\mu), \frac{m_{h}}{\mu}\right)
$$

and the $\Lambda$ parameters are related by

$$
\frac{\Lambda^{\left(N_{f}\right)}}{\Lambda^{\left(N_{f}+1\right)}}=\frac{\varphi^{\left(N_{f}\right)}\left(\bar{g}^{\left(N_{f}+1\right)} \times \xi\right)}{\varphi^{\left(N_{f}+1\right)}\left(\bar{g}^{\left(N_{f}+1\right)}\right)},
$$

where

$$
\begin{aligned}
\varphi^{\left(N_{f}\right)}(\bar{g})= & \left(b_{0} \bar{g}^{2}\right)^{-b_{1} /\left(2 b_{0}^{2}\right)} e^{-1 /\left(2 b_{0} \bar{g}^{2}\right)} \\
& \times \exp \left\{-\int_{0}^{\bar{g}} d x\left[\frac{1}{\beta(x)}+\frac{1}{b_{0} x^{3}}-\frac{b_{1}}{b_{0}^{2} x}\right]\right\}
\end{aligned}
$$

is defined in terms of the $N_{f}$-flavor $\beta$ function in the chosen scheme.

When inserting the perturbative expansions of $\xi$ and $\beta$, we choose the mass $m_{h}$ in Eq. (18) as the $\overline{\mathrm{MS}}$ mass at its own scale, $m^{*}=\bar{m}_{\overline{\mathrm{MS}}}\left(m^{*}\right)$, and set $\mu=m^{*}$. Then the one-loop term vanishes in the perturbative expansion

$$
\xi(\bar{g}, 1)=1+c_{2} \bar{g}^{4}+c_{3} \bar{g}^{6}+c_{4} \bar{g}^{8}+O\left(\bar{g}^{10}\right) .
$$

For numerical results, we use $c_{2}, c_{3}, c_{4}$ [45-49] together with the appropriate five-loop $\beta$ function $[50-54]$ to arrive at Table III. 
TABLE III. Main results of this work. $\Lambda_{\frac{(3)}{\mathrm{MS}}}$, Eq. (17), is determined nonperturbatively and used, together with the perturbative estimates of $\Lambda_{\overline{\mathrm{MS}}}^{\left(N_{f}\right)} / \Lambda \frac{(3)}{\mathrm{MS}}$, to produce all other numbers. As additional input we use the masses $m_{\text {charm }}^{*}=1.280(25) \mathrm{GeV}$, $m_{\text {bottom }}^{*}=4.180(30) \mathrm{GeV}, m_{\mathrm{Z}}=91.1876 \mathrm{GeV}$ [7], and $m_{\text {top }}^{*}=$ 165.9(2.2) GeV [55].

\begin{tabular}{lllc}
\hline \hline$N_{f}$ & $\Lambda_{\frac{\mathrm{MS}}{\left(N_{f}\right)}}^{\mathrm{MS}}[\mathrm{MeV}]$ & \multicolumn{1}{c}{$\mu$} & $\alpha_{\mathrm{MS}}^{\left(N_{f}\right)}(\mu)$ \\
\hline 4 & $298(12)(3)$ & & \\
5 & $215(10)(3)$ & $m_{Z}$ & $0.11852(80)(25)$ \\
6 & $91.1(4.5)(1.3)$ & $1.508 \mathrm{TeV}$ & $0.08523(41)(12)$ \\
\hline \hline
\end{tabular}

The first error in $\alpha(\mu)$ is due to $\Lambda_{\overline{\mathrm{MS}}}^{(3)}$ and the quark mass uncertainties, where the latter are hardly noticeable. The second error listed represents our estimate of the truncation error in PT in the connection $\Lambda_{\frac{(3)}{\mathrm{MS}}} \rightarrow \Lambda_{\mathrm{MS}}^{(4)}-\Lambda_{\overline{\mathrm{MS}}}^{(6)}$. We arrive at it as follows. The 2-, 3-, 4-loop terms in Eq. (21) combined with the 3-, 4-, 5-loop $\beta$ functions in Eq. (20) lead, e.g., to contributions $128,19,6$ in units of $10^{-5}$ to $\alpha \frac{(5)}{\mathrm{MS}}$. We take the sum of the last two contributions as our perturbative uncertainty. Within PT, this is conservative. Recently, Herren and Steinhauser [56] considered also $\mu \neq m$ in Eq. (18). Their error estimate, 0.0004, would change little in the uncertainty of our final result

$$
\alpha_{\overline{\mathrm{MS}}}^{(5)}\left(m_{Z}\right)=0.11852(84)
$$

Summary and conclusions.-QCD offers a plethora of quantities, like hadron masses and meson decay constants, that can be used as precise experimental input to compute the strong coupling and quark masses. However, the nonperturbative character of the strong interactions makes these computations difficult. Lattice QCD offers a unique tool to connect, from first principles, well-measured QCD quantities at low energies to the fundamental parameters of the standard model. As perturbative expansions are not convergent, but only asymptotic, the challenge for precise results is to nonperturbatively reach energy scales where the strong coupling is small enough [13]. Because of the slow running of $\alpha_{s}$, the hadronic and perturbative regimes are separated by 2 to 3 orders of magnitude.

Finite-size scaling allows one to bridge such large energy differences nonperturbatively. It trades the systematic uncertainties associated with the truncation of the perturbative series at relatively low energies for statistical uncertainties which are easy to estimate.

Our precise data for the running coupling [13,14], together with the high-quality set of ensembles provided by the CLS initiative [30] at lattice spacings as small as $a \approx 0.039 \mathrm{fm}$, and an accurate determination of the scale [31], allow us to reach a precision of $0.7 \%$ in $\alpha_{\overline{\mathrm{MS}}}^{(5)}\left(m_{Z}\right)$.
The factor $\mu_{\mathrm{PT}} / \mu_{\text {had }}$ contributes $87 \%$ of the uncertainty in $\alpha \frac{(5)}{\mathrm{MS}}$. This uncertainty is dominantly statistical and could certainly be reduced significantly by some additional effort. While present knowledge indicates small and perturbatively computable quark-loop effects in the matching at the heavy-quark thresholds, the uncomfortable need of using PT at scales as low as $m_{\text {charm }}$ can only be avoided by a full four-flavor computation. This is a mandatory step as soon as one attempts another controlled reduction of the total uncertainty.

We finally note, that our result $\alpha_{\frac{\mathrm{MS}}{(6)}}(1.508 \mathrm{TeV})=$ $0.0852(4)$ is in good agreement with the recent CMS determination [57] from jet cross sections with $p_{T} \in$ $[1.41,2.5] \mathrm{TeV}$. Reference [57] gives $\alpha \frac{(5)}{\mathrm{MS}}(1.508 \mathrm{TeV})=$ $0.0822(33)$ which was already converted to $\alpha \frac{(6)}{\mathrm{MS}}(1.508 \mathrm{TeV})=0.0840(35)$ in [56]. Although LHC data do not yet reach the precision of our result (evolved from lower energy), comparisons at such high energies are an excellent test of QCD and of the existence of massive colored quanta.

The technical developments which enabled the results presented in this Letter are based on seminal ideas and ground breaking work by Martin Lüscher, Peter Weisz, and Ulli Wolff, most importantly, the use of finite-size scaling methods for renormalized couplings, perturbation theory on the lattice and in the SF to two loop order, and the use of the gradient flow. We would like to express our gratitude to Martin, Peter, and Ulli for collaborative work, numerous enlightening discussions and advice over the years. Furthermore, we thank our colleagues in the ALPHA Collaboration for helpful feedback and P. Marquard and P. Uwer for discussions on the $\overline{\mathrm{MS}}$ top quark mass. We thank our colleagues in the Coordinated Lattice Simulations (CLS) effort for the joint generation of the gauge field ensembles on which the computation described here is based. We acknowledge PRACE for awarding us access to resource FERMI based in Italy at CINECA, Bologna and to resource SuperMUC based in Germany at LRZ, Munich. We are grateful for the support received by the computer centers. The authors gratefully acknowledge the Gauss Centre for Supercomputing (GCS) for providing computing time through the John von Neumann Institute for Computing (NIC) on the GCS share of the supercomputer JUQUEEN at Jülich Supercomputing Centre (JSC). GCS is the alliance of the three national supercomputing centres HLRS (Universität Stuttgart), JSC (Forschungszentrum Jülich), and LRZ (Bayerische Akademie der Wissenschaften), funded by the German Federal Ministry of Education and Research (BMBF) and the German State Ministries for Research of Baden-Württemberg (MWK), Bayern (StMWFK) and Nordrhein-Westfalen (MIWF). We thank the computer centers at HLRN (bep00040), NIC at DESY Zeuthen, and CESGA at CSIC (Spain) for providing 
computing resources and support. M. B. was supported by the U.S. D.O.E. under Grant No. DE-SC0012704. M. D. B. is grateful to CERN for the hospitality and support. S. Si. acknowledges support by SFI under Grant No. 11/ RFP/PHY3218. This work is based on previous work [18] supported strongly by the Deutsche Forschungsgemeinschaft in the SFB/TR 09.

[1] S. Dittmaier et al., arXiv:1201.3084.

[2] J. R. Andersen et al. (LHC Higgs Cross Section Working Group), arXiv:1307.1347.

[3] A. Accardi et al., Eur. Phys. J. C 76, 471 (2016).

[4] D. de Florian et al. (LHC Higgs Cross Section Working Group), arXiv:1610.07922.

[5] W. A. Bardeen, A. J. Buras, D. W. Duke, and T. Muta, Phys. Rev. D 18, 3998 (1978).

[6] W. Celmaster and R. J. Gonsalves, Phys. Rev. D 20, 1420 (1979).

[7] C. Patrignani et al. (Particle Data Group), Chin. Phys. C 40, 100001 (2016).

[8] S. Aoki et al. (PACS-CS Collaboration), J. High Energy Phys. 10 (2009) 053.

[9] M. Lüscher, P. Weisz, and U. Wolff, Nucl. Phys. B359, 221 (1991).

[10] M. Lüscher, R. Sommer, P. Weisz, and U. Wolff, Nucl. Phys. B413, 481 (1994).

[11] G. de Divitiis, R. Frezzotti, M. Guagnelli, M. Lüscher, R. Petronzio, R. Sommer, P. Weisz, and U. Wolff (ALPHA Collaboration), Nucl. Phys. B437, 447 (1995).

[12] K. Jansen, C. Liu, M. Lüscher, H. Simma, S. Sint, R. Sommer, P. Weisz, and U. Wolff, Phys. Lett. B 372, 275 (1996).

[13] M. Dalla Brida, P. Fritzsch, T. Korzec, A. Ramos, S. Sint, and R. Sommer (ALPHA Collaboration), Phys. Rev. Lett. 117, 182001 (2016).

[14] M. Dalla Brida, P. Fritzsch, T. Korzec, A. Ramos, S. Sint, and R. Sommer (ALPHA Collaboration), Phys. Rev. D 95, 014507 (2017).

[15] M. Bruno, J. Finkenrath, F. Knechtli, B. Leder, and R. Sommer (ALPHA Collaboration), Phys. Rev. Lett. 114, 102001 (2015).

[16] T. Korzec, F. Knechtli, S. Cali, B. Leder, and G. Moir, Proc. Sci., LATTICE2016 (2017) 126.

[17] S. Aoki et al., Eur. Phys. J. C 77, 112 (2017).

[18] R. Sommer and U. Wolff, Nucl. Part. Phys. Proc. 261-262, 155 (2015).

[19] M. Lüscher, R. Narayanan, P. Weisz, and U. Wolff, Nucl. Phys. B384, 168 (1992).

[20] S. Sint, Nucl. Phys. B421, 135 (1994).

[21] P. Fritzsch and A. Ramos, J. High Energy Phys. 10 (2013) 008.

[22] A. González-Arroyo, J. Jurkiewicz, and C. Korthals-Altes, Structural elements in particle physics and statistical mechanics, NATO ASI Ser., Ser. B Vol. 82, (Springer, Boston, MA, 1983), p. 339.

[23] S. Sint and P. Vilaseca, Proc. Sci., LATTICE2012, 031 (2012).

[24] A. Bode, U. Wolff, and P. Weisz (ALPHA Collaboration), Nucl. Phys. B540, 491 (1999).
[25] A. Bode, P. Weisz, and U. Wolff (ALPHA Collaboration), Nucl. Phys. B576, 517 (2000); B600, 453(E) (2001); B608, 481(E) (2001).

[26] M. Lüscher, J. High Energy Phys. 08 (2010) 071.

[27] M. Lüscher and P. Weisz, J. High Energy Phys. 02 (2011) 051.

[28] Z. Fodor, K. Holland, J. Kuti, D. Nogradi, and C. H. Wong, J. High Energy Phys. 11 (2012) 007.

[29] M. Dalla Brida, P. Fritzsch, T. Korzec, R. Ramos, S. Sint, and R. Sommer (to be published).

[30] M. Bruno et al., J. High Energy Phys. 02 (2015) 043.

[31] M. Bruno, T. Korzec, and S. Schaefer, Phys. Rev. D 95, 074504 (2017).

[32] W. Bietenholz et al., Phys. Lett. B 690, 436 (2010).

[33] See Supplemental Material at http://link.aps.org/ supplemental/10.1103/PhysRevLett.119.102001 for an overview of additional simulations and details regarding the hadronic matching interpolation of $\mu_{\mathrm{ref}}^{*} / \mu_{\text {had }}$, including tables

[34] M. Lüscher, S. Sint, R. Sommer, and P. Weisz, Nucl. Phys. B478, 365 (1996).

[35] P. Fritzsch and T. Korzec (to be published).

[36] M. Lüscher, S. Sint, R. Sommer, and H. Wittig, Nucl. Phys. B491, 344 (1997).

[37] T. Bhattacharya, R. Gupta, W. Lee, S. R. Sharpe, and J. M. Wu, Phys. Rev. D 73, 034504 (2006).

[38] S. Sint and R. Sommer, Nucl. Phys. B465, 71 (1996).

[39] M. Lüscher and S. Schaefer, Comput. Phys. Commun. 184, 519 (2013).

[40] J. Bulava and S. Schaefer, Nucl. Phys. B874, 188 (2013).

[41] openQCD—Simulation program for lattice QCD, http:// luscher.web.cern.ch/luscher/openQCD/.

[42] S. Schaefer, R. Sommer, and F. Virotta (ALPHA Collaboration), Nucl. Phys. B845, 93 (2011).

[43] U. Wolff (ALPHA Collaboration), Comput. Phys. Commun. 156, 143 (2004); Erratum: Comput. Phys. Commun. 176, 383(E) (2007).

[44] S. Weinberg, Phys. Rev. D 8, 3497 (1973).

[45] S. Weinberg, Phys. Lett. 91B, 51 (1980).

[46] W. Bernreuther and W. Wetzel, Nucl. Phys. B197, 228 (1982); Erratum: Nucl. Phys. B 513, 758(E) (1998)].

[47] A. G. Grozin, M. Höschele, J. Hoff, and M. Steinhauser, J. High Energy Phys. 09 (2011) 066.

[48] K. G. Chetyrkin, J. H. Kühn, and C. Sturm, Nucl. Phys. B744, 121 (2006)

[49] Y. Schröder and M. Steinhauser, J. High Energy Phys. 01 (2006) 051.

[50] T. van Ritbergen, J. A. M. Vermaseren, and S. A. Larin, Phys. Lett. B 400, 379 (1997).

[51] M. Czakon, Nucl. Phys. B710, 485 (2005).

[52] P. A. Baikov, K. G. Chetyrkin, and J. H. Kühn, Phys. Rev. Lett. 118, 082002 (2017).

[53] T. Luthe, A. Maier, P. Marquard, and Y. Schröder, J. High Energy Phys. 07 (2016) 127.

[54] F. Herzog, B. Ruijl, T. Ueda, J. A. M. Vermaseren, and A. Vogt, J. High Energy Phys. 02 (2017) 090.

[55] J. Fuster, A. Irles, D. Melini, P. Uwer, and M. Vos, arXiv: 1704.00540.

[56] F. Herren and M. Steinhauser, arXiv:1703.03751.

[57] V. Khachatryan et al. (CMS Collaboration), J. High Energy Phys. 03 (2017) 156. 